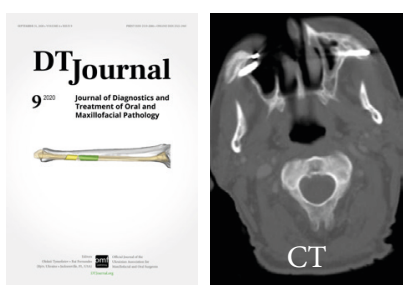

\title{
CASE
}

\section{Zygomatic Implants for Restoration of Complex Nasal Defects - A Case Report and Outcome}

\author{
John M. Le ${ }^{a, *}$, Po-Hsu Chen ${ }^{b}$ Julius C. Seidenfaden`, Anthony B. Morlandt ${ }^{\natural}$ \& Michael T. Kase
}

\section{SUMMARY}

Total rhinectomy defects pose a challenge for the reconstructive surgeon, but since the introduction of osseointegrated implants, maxillofacial implant-retained prosthetic rehabilitation has provided the patient with an alternative option that has an excellent cosmetic result. Traditionally, zygomatic implants are used for prosthodontic restoration in patients with severely atrophic maxilla or to retain an obturator after tumor ablative surgery. More recently, the nonconventional use of zygomatic implants for retention of a nasal prosthesis has been reported in cases involving rhinectomy defects where the length of conventional dental implants is a limiting factor. In this article, we describe the use and value of transversely-oriented zygomatic implants in combination with an acrylic keeper and maxillary denture to optimize retention of a complex, multi-unit prosthesis in an edentulous patient with a total rhinectomy and upper lip defect.

Birmingham, Alabama, United States

a DDS; Year 3 Resident, 6-year MD-integrated OMS Residency Program, Department of Oral and Maxillofacial Surgery, University of Alabama at Birmingham.

${ }^{\mathrm{b}}$ DDS, MS; Fellow, Department of Maxillofacial Prosthodontics, University of Alabama at Birmingham.

${ }^{c}$ CDT; Anaplastologist, Department of Maxillofacial Prosthodontics, University of Alabama at Birmingham.

${ }^{d}$ DDS, MD, FACS; Associate Professor, Department of Oral and Maxillofacial Surgery, University of Alabama at Birmingham.

e DMD; Assistant Professor, Department of Maxillofacial Prosthodontics, University of Alabama at Birmingham.

* Corresponding author's address: Department of Oral and Maxillofacial Surgery, School of Dentistry, Rm 406, 1919 7th Avenue South, Birmingham, AL 35233, United States. Phone: 562.290.7761; Fax: 205.975.6671.

E-mail:johnmtle@gmail.com (John Le)

Instagram: @surgeon_foodie
E-mails of co-authors:

pohsu@uab.edu(Po-Hsu Chen)

maxpros1@uab.edu (Julius Seidenfaden)

morlandt@uab.edu (Anthony Morlandt)

mkase@uabmc.edu (Michael Kase)

Please cite this article as: Le JM, Chen $\mathrm{PH}$, Seidenfaden JC, Morlandt AB, Kase MT. Zygomatic implants for restoration of complex nasal defects - a case report and outcome. J Diagn Treat Oral Maxillofac Pathol 2020;4(9):152-61.

Letters ' $\mathrm{CT}$ ' at the upper right icon means that article contains post-operative computed tomography (CT) data.

Paper received 22 June 2020

Accepted 25 August 2020

Available online 30 September 2020

https://dx doi.org/10.23999/j.dtomp.2020.9.2.

(C) 2020 OMF Publishing, LLC. This is an open access article under the CC BY license (http://creativecommons.org/licenses/by-nc/4.0/). 


\section{INTRODUCTION}

Extensive nasal defects due to tumor ablative surgery pose a number of reconstructive challenges. In patients with malignant pathology, local disease control may be challenging and recurrent disease can compromise previous surgical reconstruction attempts, limiting reconstructive options. In cases that involve total rhinectomy with extensive circumferential hard and soft tissue deficits, the optimal cosmetic reconstructive option may be an osseointegrated implant-retained prosthesis.

The use and value of osseointegrated implants for extraoral prosthetic rehabilitation has been increasingly advocated in the literature for maxillofacial defects. ${ }^{1-4}$ In cases of extensive atrophy or loss of the maxillary bone due to ablative surgery, the use of zygomatic implants alone or with conventional dental implants provide retention for an intraoral prosthesis. ${ }^{5,6}$ In these instances, the zygomatic implants are typically oriented obliquely, to engage the zygomatic and palatal bone, with the fixture mount surface projecting into the oral cavity. Their use for retention of nasal prosthesis following rhinectomy, where the bony components of the piriform aperture are not present or insufficient for conventional implant placement, has also been reported..$^{7-9}$ To date however, combined facial/intraoral prostheses supported by horizontally-oriented zygomatic implants have not been reported in the literature.

Here, we present an edentulous patient with an extensive rhinectomy that was restored with a nasal prosthesis retained by magnetic retention by zygomatic implants and a denture using an acrylic keeper.

\section{CASE}

A 72-year-old man was referred for treatment of recurrent basal cell carcinoma (BCC) of the right nasolabial fold. He had previously undergone multiple wide local excisions with local flap reconstruction for a primary and recurrent $\mathrm{BCC}$ of the right nasal ala that ultimately resulted in a defect comprising the majority of the nasal complex, bilateral nasal cavity, and left medial canthus along with the lacrimal system. Due to the aggressive nature of the disease, the patient was discussed at the multidisciplinary tumor board where it was agreed that the best option for cure included total rhinectomy, infrastructure maxillectomy, and excision of the involved upper lip. Due the extent of the anticipated defect and the need for adjuvant radiotherapy, osseointegrated implant-retained maxillofacial prosthesis was felt to represent the best reconstructive option for the patient. To minimize the risk of osteoradionecrosis, implants were placed at the time of ablative surgery, 6-8 weeks prior to starting adjuvant radiation therapy.

Following tumor extirpation, there was a large facial defect that involved the nasal bones, right infraorbital rim and lacrimal bone, right cheek skin, upper lip, right maxilla sinus, inferior turbinate, and anterior maxilla. Two horizontallyoriented zygomatic implants $(47.5-\mathrm{mm}$ left and $37.5-\mathrm{mm}$ right) were then placed into the remaining maxillae and zygomae bilaterally (Bränemark System; Nobel Biocare ${ }^{\mathrm{m} x}$, Zürich, Switzerland) (Fig 1).

The patient then underwent 60 Gy of adjuvant radiotherapy in 30 fractions without complication. At the initiation of the prosthetic phase of treatment, the defect site was well-healed, and the zygomatic implants were stable without evidence of mobility. The key component that optimized the retention of the nasal prosthesis was the fabrication of an acrylic keeper that was incorporated with two magnets to match the nasal prosthesis and upper denture (Figs 2 and 3 ).

Fifteen months after the completion of radiotherapy, the patient remained free of disease and the nasal prosthesis along with the dentures was in function, with an excellent cosmetic outcome and no evidence of implant or prosthetic failure (Figs 4-6).

\section{DISCUSSION}

Total rhinectomy with extensive adjacent hard and soft tissue involvement can be a challenge for the reconstructive surgeon. In cases with malignant pathology, there is a risk for recurrence, and additional adjuvant radiotherapy may be indicated. Because of these factors, a horizontally oriented, zygomatic implant-retained maxillofacial prosthesis can optimize treatment, provide a superior cosmetic outcome and patient satisfaction compared to local or free flap reconstruction. 

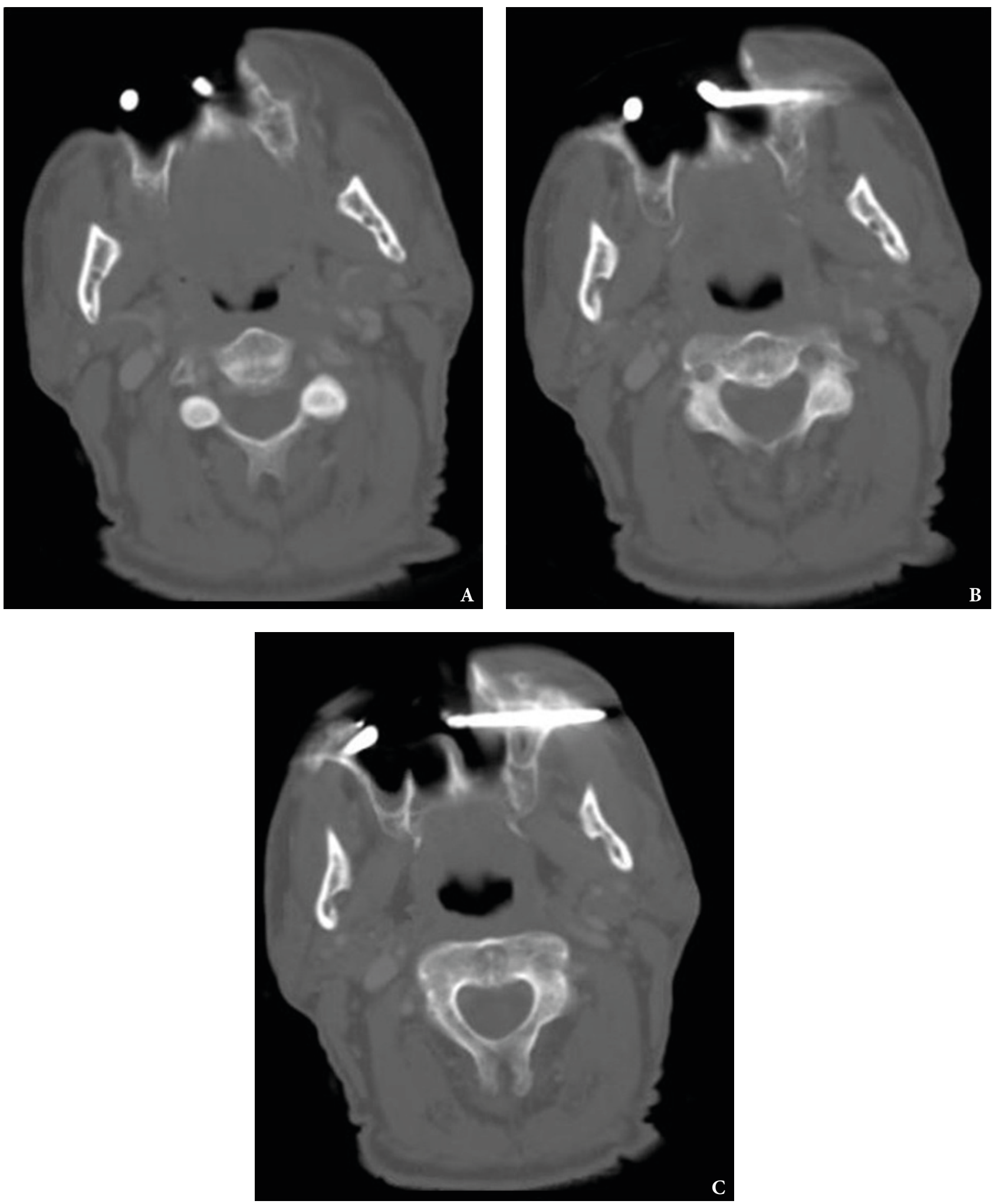

FIGURE 1. Serial computed tomography (CT) imaging (axial view) of zygomatic implants. Where $\mathbf{A}$ is the most caudal CT scan and $\mathbf{F}$ is the most cranial one. (Fig 1 continued on next page.) 

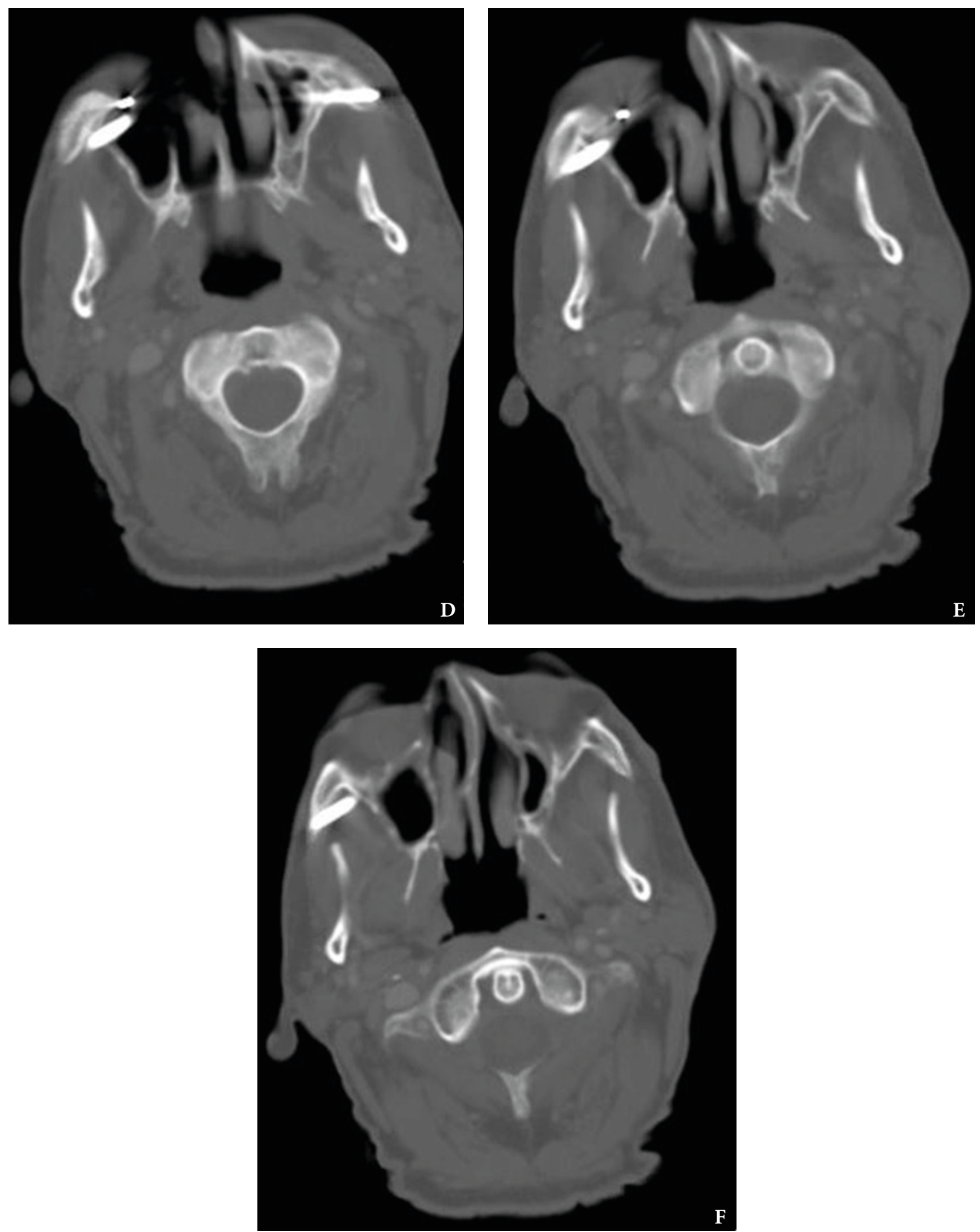

FIGURE 1 (cont'd). Serial computed tomography (CT) imaging (axial view) of zygomatic implants. Where $\mathbf{A}$ is the most caudal CT scan and $\mathbf{F}$ is the most cranial one. 


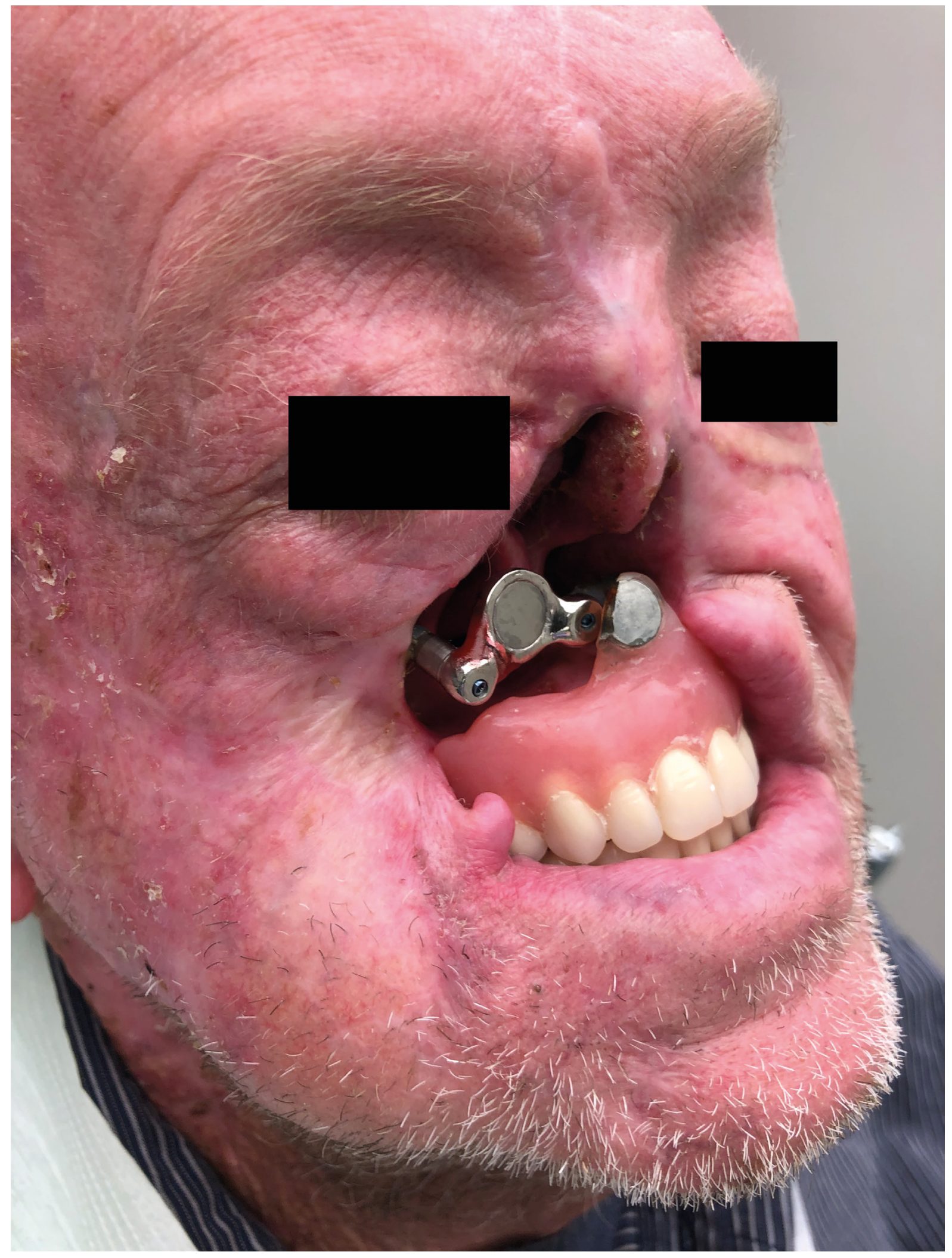

FIGURE 2. Three-quarter view with upper denture in place. 


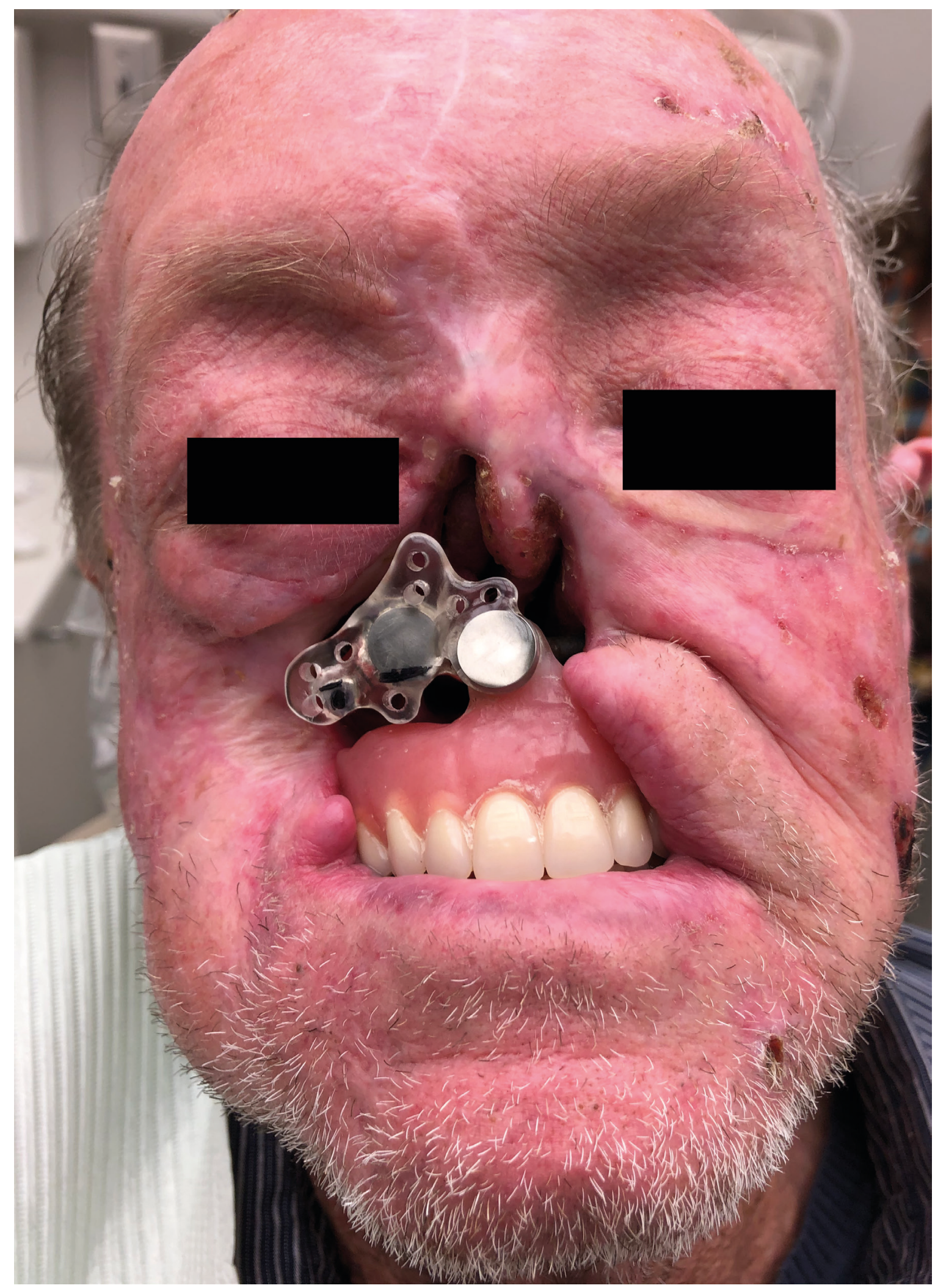

FIGURE 3. Frontal view with acrylic keeper connecting maxillary denture to zygomatic implant connector. 


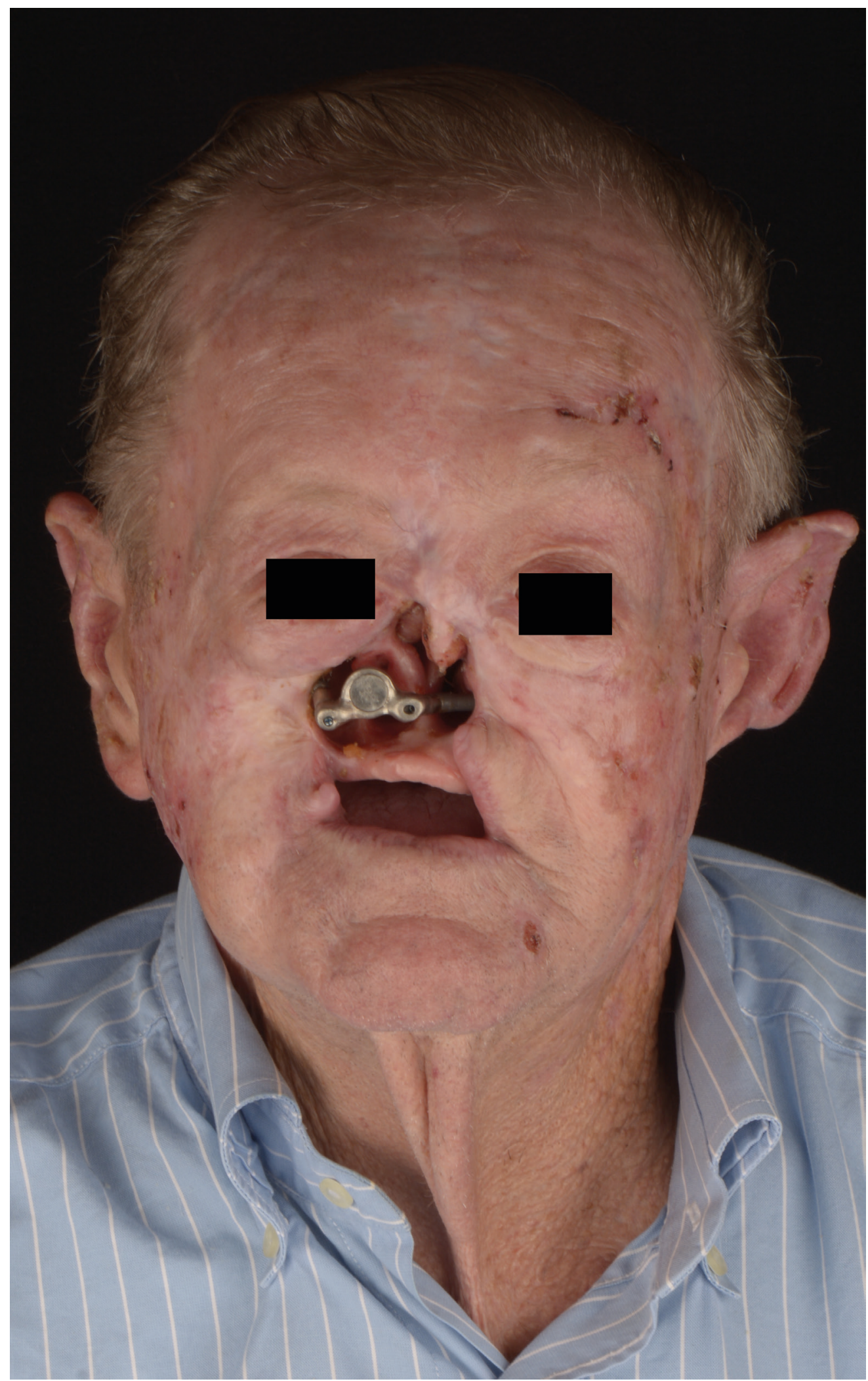

FIGURE 4. Postoperative frontal view with zygomatic implants attached to magnetic component for the nasal prosthesis. 


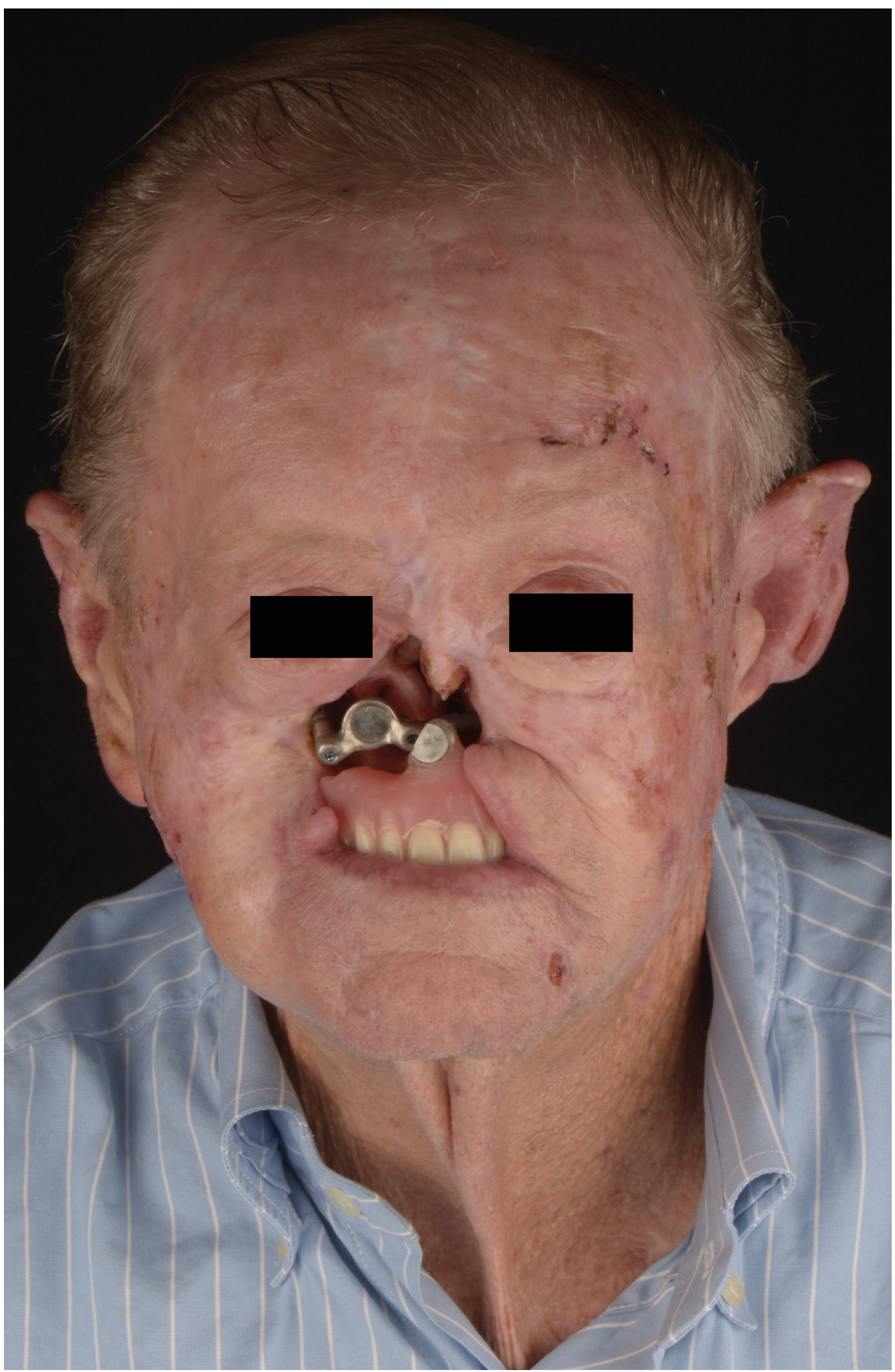

FIGURE 5. Postoperative frontal view with the upper complete denture in place with a magnet component attached to the anterior superior flange for the nasal prosthesis. 


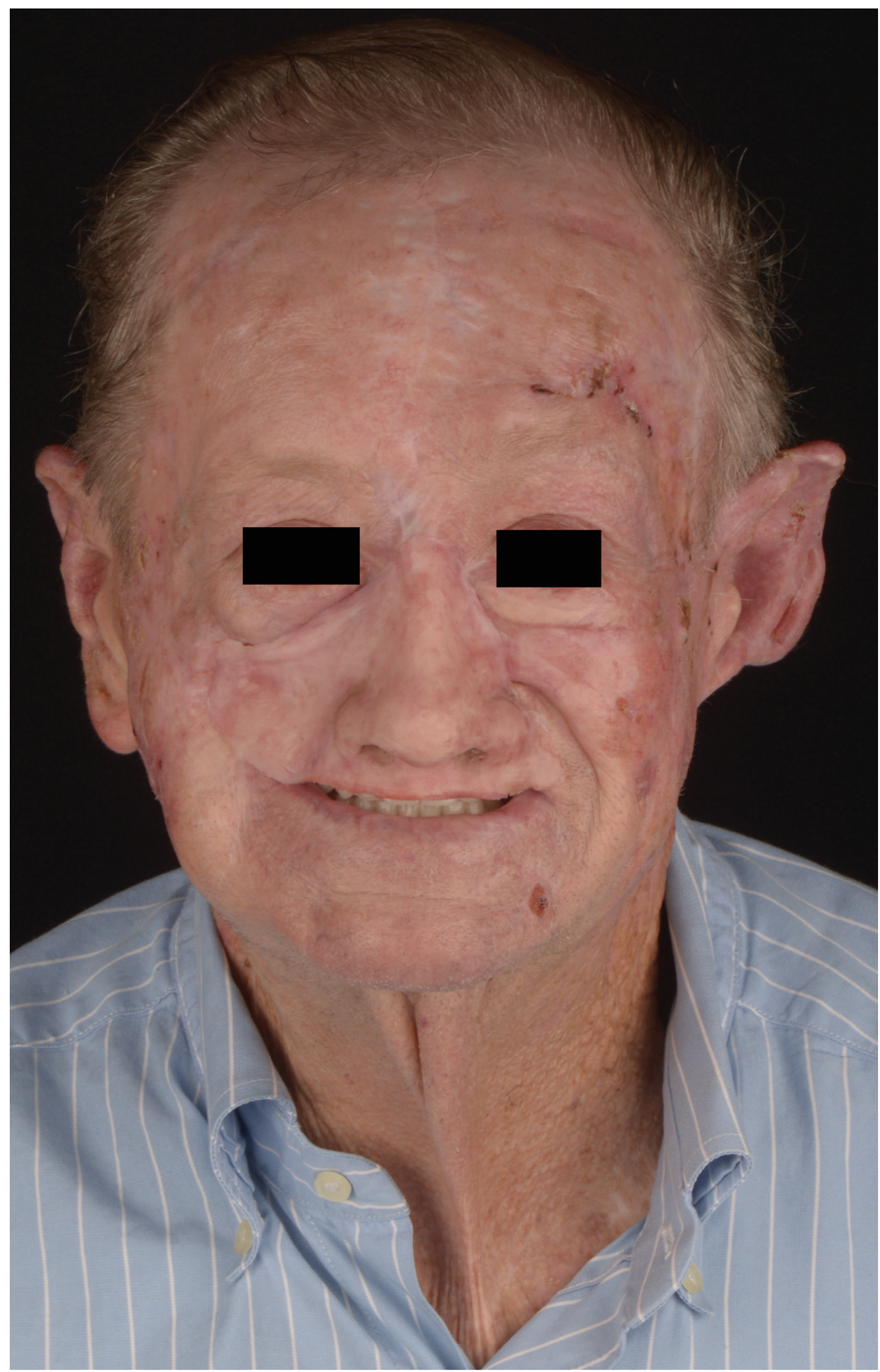

FIGURE 6. Frontal view with the final nasal prosthesis and complete dentures in place. 
Although the risks for implant failure and infection exist, as well as the need for long-term prosthetic maintenance, the success rate of zygomatic implant-retained facial prosthesis remains high..$^{9-10}$ Furthermore, the area of the disease can be easily surveilled by the oncologic surgeon.

In our case, we demonstrate the successful outcome of a zygomatic implant-retained nasal prosthesis in an edentulous patient with an extensive nasal defect involving the maxilla, right cheek, and upper lip. While the proper retention of a conventional complete denture was not achievable in our patient due to the compromised anatomical structure at the anterior denture border, by applying an acrylic keeper to connect the magnetic components of the maxillary denture and nasal prosthesis together with the zygomatic implants, the retention of the maxillofacial prosthesis was optimized. The patient was free of disease at the 15 -month mark and very pleased with the intraand extraoral prostheses.

\section{CONFLICT OF INTEREST}

No conflict of interest.

\section{FUNDING}

This study did not receive any funding source.

\section{ROLE OF CO-AUTHORS IN WRITING}

All authors contributed equally to the concept and design of the study; writing, editing, and final review of the manuscript. The material collection and data processing was completed by the first author.

\section{PATIENT CONSENT}

Consent was obtained by the patient in writing and verbally for publication of photos.

\section{REFERENCES}

1. Tolman DE, Desjardins RP. Extraoral application of osseointegrated implants. J Oral Maxillofac Surg 1991;49(1):33-45. https://doi.org/10.1016/02782391(91)90264-m.

2. Ueda M, Hibino Y, Niimi A. Usefulness of dental implants in maxillofacial reconstruction. J Long Term Eff Med Implants 1999;9(4):349-66.

3. Roumanas ED, Chang TL, Beumer J. Use of osseointegrated implants in the restoration of head and neck defects. J Calif Dent Assoc 2006;34(9):711-8.

4. Flood TR, Russell K. Reconstruction of nasal defects with implant-retained nasal prostheses. $\mathrm{Br} \mathrm{J}$ Oral Maxillofac Surg 1998;36(5):341-5. https://doi. org/10.1016/s0266-4356(98)90644-1.

5. Aparicio C, Manresa C, Francisco K, Claros P, Alández J, González-Martín O, Albrektsson T. Zygomatic implants: indications, techniques and outcomes, and the zygomatic success code. Periodontol 2000 2014;66(1):41-58. https://doi.org/10.1111/cid.12047.

6. Gómez-Pedraza A, González-Cardín V, Díez-Suárez L, Herrera-Villalva M. Maxillofacial rehabilitation with zygomatic implants in an oncologic patient: a case report. J Oral Maxillofac Surg 2020;78(4):54756. https://doi.org/10.1016/j.joms.2019.10.006.

7. Bowden JR, Flood TR, Downie IP. Zygomaticus implants for retention of nasal prostheses after rhinectomy. Br J Oral Maxillofac Surg 2006;44(1):546. https://doi.org/10.1016/j.bjoms.2005.04.006.

8. Ethunandan M, Downie I, Flood T. Implantretained nasal prosthesis for reconstruction of large rhinectomy defects: the Salisbury experience. Int $J$ Oral Maxillofac Surg 2010;39(4):343-9. https://doi. org/10.1016/j.ijom.2010.01.003.

9. Scott N, Kittur MA, Evans PL, Dovgalski L, Hodder SC. The use of zygomatic implants for the retention of nasal prosthesis following rhinectomy: the Morriston experience. Int J Oral Maxillofac Surg 2016;45(8):10448. https://doi.org/10.1016/j.ijom.2016.01.020.

10. Goiato MC, Pesqueira AA, da Silva CR, Gennari Filho $\mathrm{H}$, Micheline Dos Santos D. Patient satisfaction with maxillofacial prosthesis. Literature review. J Plast Reconstr Aesthet Surg 2009;62(2):175-80. https://doi. org/10.1016/j.bjps.2008.06.084. 